\title{
BMJ Open Incidence, duration and cost of futile treatment in end-of-life hospital admissions to three Australian public- sector tertiary hospitals: a retrospective multicentre cohort study
}

\author{
Hannah E Carter, ${ }^{1}$ Sarah Winch, ${ }^{2}$ Adrian G Barnett, ${ }^{1}$ Malcolm Parker, ${ }^{2}$ \\ Cindy Gallois, ${ }^{3}$ Lindy Willmott, ${ }^{4}$ Ben P White, ${ }^{4}$ Mary Anne Patton, ${ }^{2}$ Letitia Burridge,${ }^{2}$ \\ Gayle Salkield, ${ }^{2}$ Eliana Close,${ }^{4}$ Leonie Callaway, ${ }^{2}$ Nicholas Graves ${ }^{1}$
}

To cite: Carter HE, Winch S, Barnett AG, et al. Incidence, duration and cost of futile treatment in end-of-life hospital admissions to three Australian public-sector tertiary hospitals: a retrospective multicentre cohort study. BMJ Open 2017;7:e017661. doi:10.1136/ bmjopen-2017-017661

- Prepublication history and additional material for this paper are available online. To view, please visit the journal (http:// dx.doi.org/10.1136/bmjopen2017-017661).

Received 12 May 2017 Revised 19 July 2017

Accepted 31 July 2017

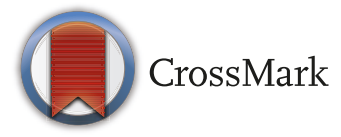

${ }^{1}$ Australian Centre for Health Services Innovation, Queensland University of Technology, Brisbane, Australia

${ }^{2}$ Faculty of Medicine, The University of Queensland, Brisbane, Australia

${ }^{3}$ School of Psychology, The University of Queensland,

Brisbane, Australia

${ }^{4}$ Australian Centre for Health Law Research, Queensland University of Technology, Brisbane, Australia

Correspondence to Nicholas Graves; n.graves@qut.edu.au

\section{ABSTRACT}

Objectives To estimate the incidence, duration and cost of futile treatment for end-of-life hospital admissions.

Design Retrospective multicentre cohort study involving a clinical audit of hospital admissions.

Setting Three Australian public-sector tertiary hospitals. Participants Adult patients who died while admitted to one of the study hospitals over a 6-month period in 2012. Main outcome measures Incidences of futile treatment among end-of-life admissions; length of stay in both ward and intensive care settings for the duration that patients received futile treatments; health system costs associated with futile treatments; monetary valuation of bed days associated with futile treatment.

Results The incidence rate of futile treatment in endof-life admissions was $12.1 \%$ across the three study hospitals (range 6.0\%-19.6\%). For admissions involving futile treatment, the mean length of stay following the onset of futile treatment was 15 days, with 5.25 of these days in the intensive care unit. The cost associated with futile bed days was estimated to be \$AA12.4 million for the three study hospitals using health system costs, and $\$$ A988 000 when using a decision maker's willingness to pay for bed days. This was extrapolated to an annual national health system cost of \$A153.1 million and a decision maker's willingness to pay of $\$ A 12.3$ million. Conclusions The incidence rate and cost of futile treatment in end-of-life admissions varied between hospitals. The overall impact was substantial in terms of both the bed days and cost incurred. An increased awareness of these economic costs may generate support for interventions designed to reduce futile treatments. We did not include emotional hardship or pain and suffering, which represent additional costs.

\section{INTRODUCTION}

Advances in medical technology allow clinicians in acute hospitals to save lives and lengthen the time to death. Some interventions have little chance of conferring a meaningful benefit to the patient. ${ }^{1}$ While a
Strengths and limitations of this study

- This is the first attempt to estimate the costs associated with futile treatment across a whole of hospital setting.

- We articulated the process for making determinations of futile treatment judgements yet these are inherently value-laden and subjective.

- The retrospective nature of the review process also had the potential to produce bias in clinical judgements.

- The costs reported are likely to be an upper bound as we have no knowledge of the costs of other treatments that would have happened as futile treatment not occurred.

- Increased awareness of the extent of futile treatment and its impacts should stimulate action to reduce the problem.

value-laden and contested term, such treatments are often referred to as 'futile' ${ }^{23}$ and more recently as "potentially inappropriate' or 'non-beneficial'. ${ }^{5}$ There is evidence, that for various reasons, doctors provide treatments they perceive as futile. ${ }^{6-8}$ These can prevent patients from experiencing a good death, cause distress to family members and medical staff and use up scarce resources. ${ }^{9}$ Studies limited to paediatric or adult intensive care settings have investigated the relationship between hospital administered futile treatment and resource use. ${ }^{10}{ }^{11}$ Information on the cost of futile treatment that occurs across the broader hospital setting for patients at the end of life is unavailable. Futile treatments in many cases will be an inappropriate use of scarce healthcare resources and so data about the frequency and magnitude of this problem is valuable for decision makers in 
both the hospital and broader healthcare setting. It may stimulate interventions designed to reduce its frequency. The aims of this study are to estimate the incidence and duration of futile treatment in end-of-life hospital admissions and to assign a monetary value to the hospital bed days that were used.

\section{METHOD}

A retrospective cohort study was used to identify cases of futile treatment among 907 consecutive adult admissions to three tertiary referral hospitals in Australia. Every eligible admission that ended in death and occurred during the 6 months between March and September 2012 was included. At one hospital, there was 1 month where no charts were available for review. No sample size calculation was undertaken, rather we judged this time frame sufficient to access enough information to meet the aims of the analysis.

Admissions were sourced from the medical records of the study hospitals. Patients aged under 18 years were excluded, as were patients declared dead on arrival, even if they were placed on life support to facilitate organ donation. We excluded information that would identify the hospitals. Multicentre ethics approval for the study was obtained for all the relevant hospitals and universities. Access to patients' medical records was granted by the state health department.

\section{Identifying futile treatment}

The assessment of futile treatment emerged from four consecutive steps, consisting of an initial nurse-led medical chart audit followed by three rounds of review by senior medical staff. An overview of this process is shown in figure 1 .

Two registered nurses were trained for the task and reviewed medical charts from all 907 end-of-life admissions at the three hospitals. This nurse audit was guided by the Brisbane Futility Audit Tool, a 47-item instrument developed using the Supportive and Palliative Care Indicators Tool criteria ${ }^{12}$ and from a review panel of experienced clinicians and researchers in end-of-life care. A copy of the audit tool is included in (online supplementary appendix 1). Inter-rater and intra-rater consistency in

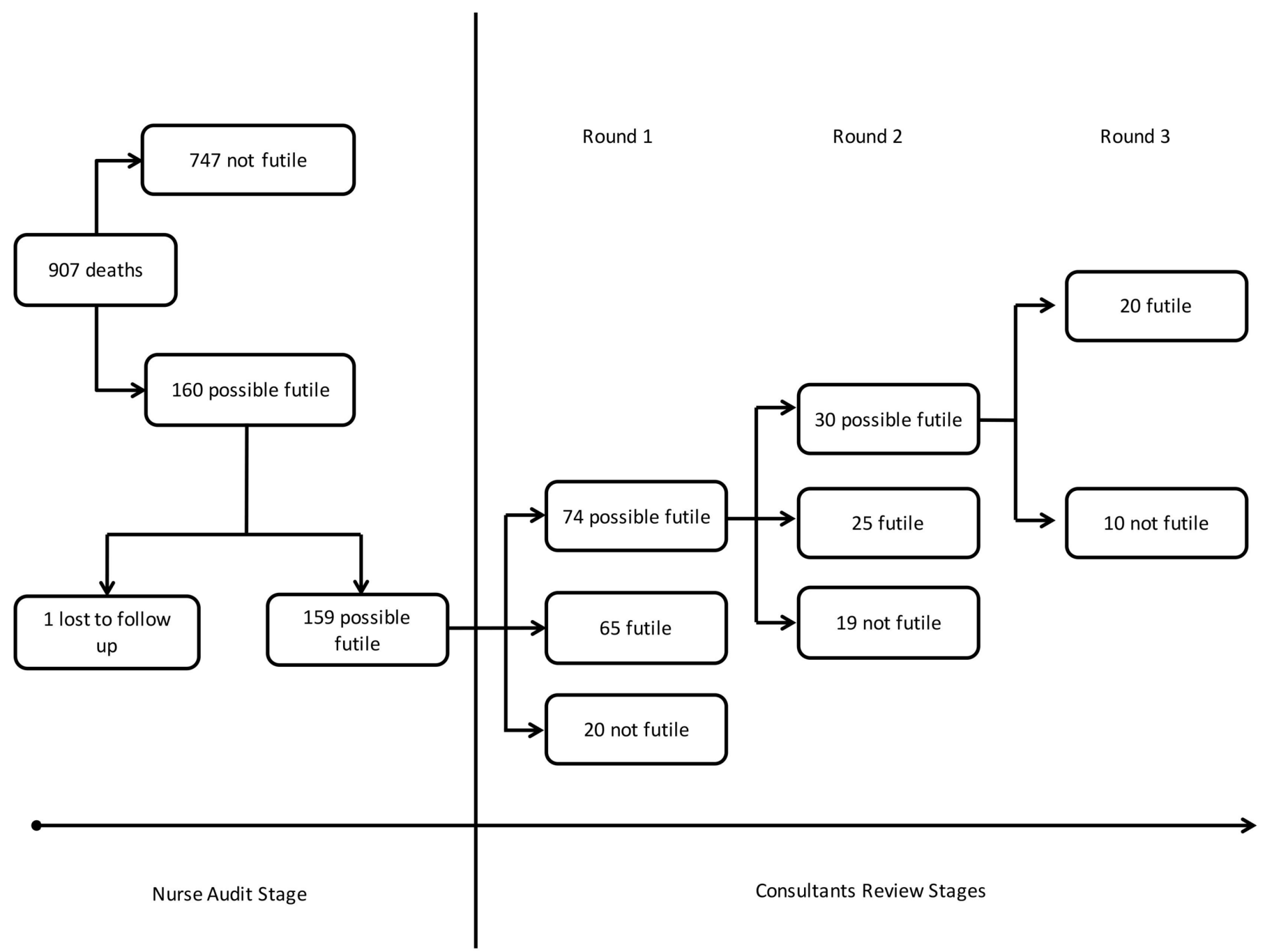

Figure 1 Processes used to judge whether futile treatment occurred during the final admission of 907 patients. Only those admissions judged as 'potentially futile' were carried forward to additional review rounds. 
the application of the tool was ascertained and confirmed after every 200 cases reviewed.

The nurse audit classified each admission as receiving treatment prior to death that was 'potentially futile' or 'not futile'. The nurses' judgements were based on this definition:

Futile treatment is treatment that does not bring benefit to the patient in terms of: improving the patient's quality of life; significantly prolonging the patient's life of acceptable quality; or involving burden that outweighs benefit.

This definition was synthesised from semistructured interviews with doctors from the three study hospitals and further detail regarding this component of the study is reported in a previous publication. ${ }^{1}$ The research nurses also rated how confident they were about this judgement on a scale of $0 \%-100 \%$. Cases where the nurses were more than $70 \%$ confident that no futile treatment was provided were screened out at this point; the remainder were classified as potentially futile.

The remaining 159 potentially futile cases were classified by consensus. Hospital-based doctors with experience in end-of-life care from the three study hospitals were invited to participate in this process. A total of 55 consultants were involved from a range of specialties including emergency medicine, internal medicine, geriatric medicine, oncology, cardiology, surgery, palliative care, renal medicine, endocrinology, intensive care, neurology, haematology, respiratory medicine and psychiatry.

Round 1 of the consensus process involved reviewing a detailed summary of each potentially futile case. Each case summary was de-identified and given a code number, then conditionally randomised to exclude cases from each consultant's own hospital. Each case was then assigned to five eligible consultants. Cases were assigned so that no two reviewers had more than 10 cases in common. Each consultant reviewed up to 25 cases using scoresheets containing instructions and the definition of futile treatment used by the nurse auditors; this is shown in (online supplementary appendix 2). Consultant reviewers were required to independently classify cases as involving treatment that was futile or not futile, and when four out of five $(80 \%)$ consultants agreed on the judgement regarding futility, it was deemed as resolved. For cases identified as futile, reviewers were asked to indicate the date that they believed the futile treatment commenced. This yielded several different nominated dates in many cases. For Round 2, the 74 cases that had failed to achieve an $80 \%$ consensus were randomly assigned to a further five consultants repeating the procedure described above. A combined minimum consensus of $60 \%$ per case across the first two rounds was required to finalise a judgement on treatment futility. The 30 remaining cases that failed to achieve $60 \%$ consensus were referred to Round 3 . This comprised three face-to-face panels of approximately five consultants who discussed each case until a final determination was made.

\section{Incidences, length of stay and cost of futile treatment}

The incidence rate of futile treatment within each hospital was calculated as the number of admissions involving futile treatment as determined by the review process, as a proportion of the total number of end-of-life admissions for the 6 months between March and September 2012.

The length of stay after futile treatment commenced was estimated as the number of days in a hospital bed until the date of death in hospital. Due to variation among the start dates specified by the different consultants that reviewed each admission, we assumed that futile treatment began on the mean number of days postadmission for all reported dates. Using the earliest date would lengthen the duration of futile treatment and using the latest date would shorten it. A sensitivity analysis was used to explore the impact of adopting the earliest and latest dates on lengths of stay and cost outcomes. Days spent receiving futile treatment were either in medical wards or the intensive care unit (ICU).

A mean cost per bed day was estimated using accounting values, where the annual operating expenditure of Australian public hospitals was divided by the number of annual patient bed days. This figure was then adjusted to reflect the relative cost of bed days occurring in the ward and ICU, with the ICU cost based on the estimate derived by Rechner and Lipman. ${ }^{13}$ All costs were then inflated to 2016 Australian prices using a national inflation index specific to medical and hospital services. ${ }^{14} \mathrm{~A}$ resulting cost of \$A2351 and \$A6141 was found for each ward and ICU bed day, respectively. Cost calculations are summarised in table 1 .

The accounting cost of a bed day reflects historical spending by health services. Hospital decision-makers thinking prospectively may not value bed days in this way. Thus an alternate approach was used, where bed days were valued in terms of a hospital Chief Executive Officer's (CEO) willingness to pay for them. This method provides an indication of a bed day's value in achieving desired hospital outcomes, often referred to as the economic opportunity cost. ${ }^{15}$ The willingness to pay estimates were informed by a 2017 study by Page $e t$ $a l^{16}$ and $\$ A 216$ was used for a ward day and \$A436 for an ICU day. We assumed that while the accounting method represented a societal perspective on the costs of futile treatment, the willingness to pay of hospital CEOs represented the perspective of hospital decision makers who might choose programmes in the future to reduce futile treatments.

To estimate the expected costs associated with futile treatment on a national level, we extrapolated by assuming the average incidence and length of stay associated with futile treatment among the three study hospitals was representative of other major Australian public hospitals. After excluding children's hospitals, we defined major Australian hospitals as those with an ICU accredited for advanced clinician training by the College of Intensive Care Medicine, and a public hospital classified by the National Health Performance Authority as a 'Major 
Table 1 Hospital bed day costing items in Australian public hospitals

\begin{tabular}{|c|c|c|c|}
\hline Item & Estimate & Source & Date \\
\hline National public hospital expenditure & $\$ A 44435000000$ & $\mathrm{AlHW}^{22}$ & 2014 \\
\hline National public hospital patient bed days & 18267000 days & $\mathrm{AlHW}^{23}$ & 2014 \\
\hline Ward days & 17875000 days & $\mathrm{AlHW}^{23}$ & 2014 \\
\hline $\begin{array}{l}\text { Average national cost per hospital bed day } \\
\text { (ward and ICU combined) }\end{array}$ & $\$ A 2433$ & Calculation* & 2014 \\
\hline CPI inflator (medical and hospital services) & $2.3 \%$ & $\mathrm{ABS}^{14}$ & 2002-2016 \\
\hline Cost per ICU bed day in 2016 & $\$ A 6141$ & Calculation $†$ & 2016 \\
\hline Cost per ward day in 2016 & $\$ A 2351$ & Calculation $\neq$ & 2016 \\
\hline
\end{tabular}

*Total public hospital expenditure divided by the total public hospital bed days. †Cost per ICU bed day in 2002 multiplied by the inflation factor.

$\ddagger$ Total public hospital expenditure, less expenditure on ICU days (applying the 2016 ICU bed day cost calculated in the second footnote), divided by annual public hospital ward days.

ABS, Australian Bureau of Statistics; AlHW, Australian Institute of Health and Welfare; CPI, Consumer Price Index; ICU, intensive care unit.

Hospital'. The full list of hospitals is in online supplementary appendix 3.

To allow for the uncertainty in the estimates statistical distributions were fitted to the data. We chose a Beta distribution to represent the incidence of futile treatment across the three hospitals, as this distribution is a good fit to the binomial distribution parameters, is restricted to the interval $0-1$ and is continuous. Gamma distributions were used for lengths of stay, as they are positive and right skewed. To generate results that show uncertainty, the distributions were randomly sampled 1000 times using simulation. The parameters for the distributions were derived from the observed data from the three hospitals. Fixed values were applied to the bed day costs assigned to each sample; $95 \%$ uncertainty intervals around the means were derived from 1000 simulations. There was no patient involvement in this research.

\section{RESULTS}

At the end of the review process, 110 of the total 907 end-of-life admissions (12.1\%) among the three hospitals involved futile treatment. The lowest mean incidence rate of futile treatment was at hospital A $(6 \%)$ relative to hospitals B $(12.8 \%)$ and C $(19.6 \%)$. The distribution of the incidences of futile treatment across the hospitals after accounting for uncertainty is shown in figure 2.

Beta distributions are used to reflect the uncertainty around the mean incidence of futile treatment. The $\mathrm{X}$-axis is the incidence of futile treatment across the distribution. The Y-axis is the number of samples from the Beta distribution that produced each incidence rate. A total of 1000 samples were generated for each hospital.

For admissions involving futile treatment, the mean length of stay following the onset of futile treatment across all three hospitals was 15.1 days. This consisted of 9.8 days spent in a ward and 5.3 days in the ICU. When examining the relative frequency of days spent receiving futile treatment across the distribution (figure 3), over $50 \%$ of admissions containing futile treatment were associated with three or fewer futile bed days. This reflects the nature of hospital admissions data where a relatively small number of admissions with long lengths of stay create an average length of stay that is higher than most patients. ${ }^{17}$

The Gamma distribution was used to reflect the uncertainty around the mean length of stay. The $\mathrm{X}$-axis is the number of days spent receiving futile treatment across the distribution. The Y-axis is the number of samples from the Gamma distribution that produced each length of stay. A total of 1000 samples were generated.

The mean lengths of stay for receiving futile treatment were similar in hospitals A (12 days) and B (12.7 days), but higher in hospital C (19.4 days) (figure 4). The number of ICU days as a proportion of the total futile length of stay ranged from $30 \%$ in hospital A to $39 \%$ in hospital C.

When results were generalised to a year, a total of 3313 bed days were associated with futile treatment across the

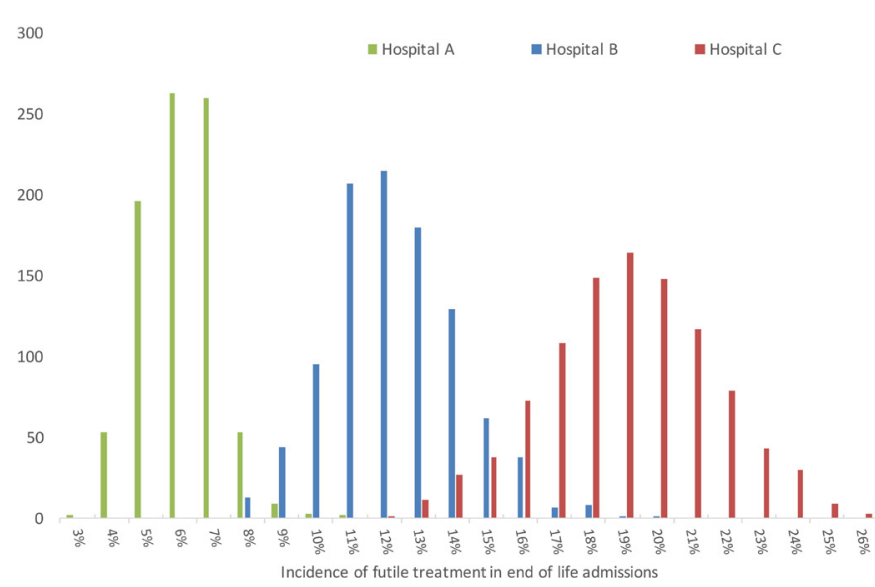

Figure 2 Incidence of futile treatment in end-of-life admissions for the three hospitals. 
600

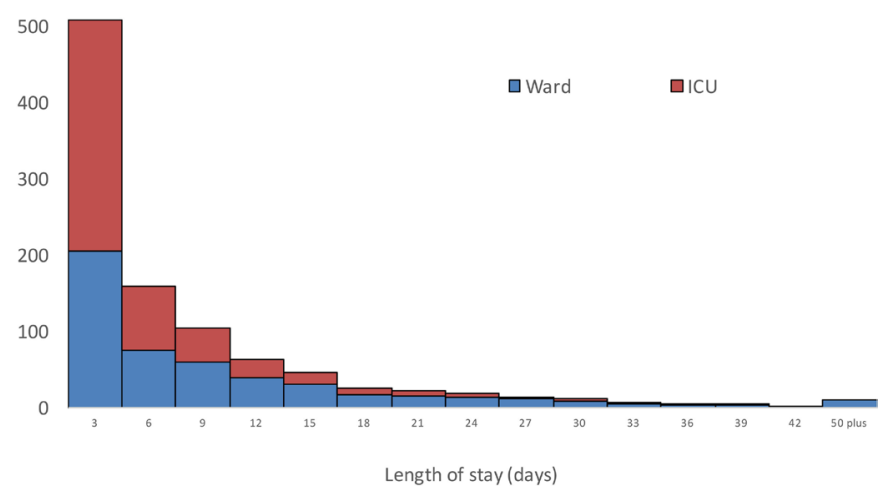

Figure 3 Length of stay while receiving futile treatment across the three study hospitals combined. ICU, intensive care unit.

combined study hospitals, with approximately $35 \%$ of these occurring in the ICU (table 2). When accounting costs were attributed to both ward and ICU days, the estimated total health system cost was $\$ \mathrm{~A} 12.4$ million across the three hospitals. The estimated willingness to pay by hospital CEOs for the bed days used for futile treatment was $\$$ A988 000 .

When extrapolated to reflect the national impact of futile treatment in major tertiary hospitals, an estimated 41222 bed days per year were attributed to futile treatment. This translated to an annual national health system cost of \$A153.1 million and a hospital willingness to pay of $\$$ A12.3 million.

A sensitivity analysis to test the date at which futile treatment was estimated to have begun is shown (figure 5). The earliest and latest dates recorded by all clinicians to have reviewed each futile case were tested. When the earliest date for futile treatment was applied, a total of 4586 bed days were attributed to futile treatment (2997 in the ward and 1529 in the ICU), translating to a total cost of $\$ \mathrm{~A} 16.4$ million and willingness to pay of $\$ \mathrm{~A} 1.3$ million. This reduced to 2035 when the latest dates were applied

25

$20 \quad$ Ward $\quad$ ICU

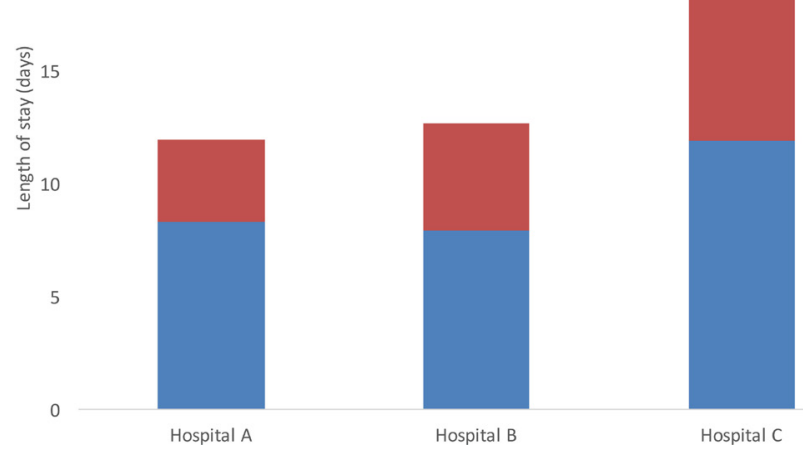

Figure 4 Mean length of stay while receiving futile treatment, by hospital. ICU, intensive care unit.
(1291 in the ward and 745 in the ICU) at a cost of $\$ \mathrm{~A} 7.6$ million and willingness to pay of $\$ \mathrm{~A} 604000$.

\section{DISCUSSION}

Estimates of the total number of hospital bed days lost to futile treatment in end-of-life admissions across three major tertiary hospitals are reported, and valued in monetary terms that reflect both the societal and hospital decision-maker perspectives. Futile treatment was associated with a total of 3313 bed days per year, translating to a value of $\$ A 12.1$ million to the health system and \$A988 000 to hospital decision makers.

This is the first attempt to estimate the costs associated with futile treatment across a whole of hospital setting. We found that both the incidence of futile treatment and the length of stay attributable to futile treatment varied between hospitals. Hospital $\mathrm{C}$ was associated with the highest incidence of futile treatment, as well as the longest average length of stay following the onset of futile treatment. Hospital A was found to have both the lowest levels of futile treatment and the shortest associated length of stay. The reasons for these differences are not known. While all three hospitals were similar in their geographic location and accredited training status, there nonetheless may be differences in the admitted patient cohorts and clinician preferences that drive treatment decisions. A 2016 study in an earlier phase of this project identified a number of hospital-specific factors that may contribute to the provision of futile treatment, including the degree of specialisation, the availability of routine tests and interventions, and organisational barriers to diverting a patient from a curative to a palliative pathway. ${ }^{18}$ It would be useful to recruit more hospitals and repeat this work to see if rates were higher or lower than those seen in hospitals A (6\%) and C (19.6\%), respectively.

Our estimates of the costs associated with futile treatment are dependent on the perspective taken. When a societal perspective was adopted, in which the cost per hospital bed day was derived as a hospital's total operating expenses per patient bed day, total costs were more than 12 times higher than what hospital CEOs would be willing to pay to free up that day. This may reflect hospital funding arrangements in Australia, where hospitals receive funding allocations up to a specified level of activity. It also may reflect the 'fixed' nature of many hospital cost items, such as permanent staffing and building overheads, that are fixed regardless of hospital activity. We suggest that the societal perspective provides a more accurate picture of the total costs of futile treatments to the healthcare system as well as an incentive to drive system-wide change. Nonetheless, a CEO's willingness to pay is likely to be an important consideration in decision making processes regarding changes to hospital-specific policies or practices.

Our research method has limitations. Although we articulated the process for making determinations of futile treatment, judgements such as these are inherently 
Table 2 Total bed days and costs associated with futile treatment over 12 months

\begin{tabular}{lllll}
\hline Item & $\begin{array}{l}\text { Hosp A } \\
(\mathbf{n = 3 3 3})\end{array}$ & $\begin{array}{l}\text { Hosp B } \\
(\mathbf{n = 3 2 4 )}\end{array}$ & $\begin{array}{l}\text { Hosp C } \\
(\mathbf{n = 2 5 0 )}\end{array}$ & $\begin{array}{l}\text { All } \\
(\mathbf{n}=\mathbf{9 0 7})\end{array}$ \\
\hline Annual ward days & 328 & 650 & 1141 & 2160 \\
\hline $95 \%$ uncertainty interval & $297-359$ & $600-703$ & $1075-1029$ & $2029-2318$ \\
\hline Annual ICU days & 143 & 382 & 716 & 1153 \\
\hline $95 \%$ uncertainty interval & $132-155$ & $358-406$ & $677-758$ & $1074-1230$ \\
\hline Annual cost to the health system (\$A thou) & 1671 & 3923 & 7008 & 12350 \\
\hline $95 \%$ uncertainty interval & $1577-1762$ & $3716-4126$ & $6709-7352$ & $11759-12954$ \\
\hline Annual hospital willingness to pay (\$A thou) & 135 & 310 & 554 & 988 \\
\hline $95 \%$ uncertainty interval & $128-143$ & $293-327$ & $529-580$ & $942-1037$ \\
\hline
\end{tabular}

value-laden and subjective. Deciding when treatment becomes futile, in many instances, requires the perspectives of patients and family members as well as multiple clinicians. The retrospective nature of the review process also had the potential to produce bias in clinical judgements. For example, the knowledge that a particular medical intervention was unsuccessful may have influenced an assessment that the intervention was futile, when such an assessment may not have been reasonably apparent in real time. A prospective randomised study of some intervention to reduce futile treatment might assemble evidence of futile treatment in real time and then be used for an audit and feedback process. The outcomes for comparison might be number of referrals to palliative care and length of stay in an acute bed. Our focus on hospital admissions ending in death ignored the potential for hospital-administered futile treatment to occur in cases where patients were discharged and later died in a hospice, residential care, or home setting. In addition, while futile treatment may also occur in a non-hospital setting, this was beyond the scope of the study. As such, our results describe futile treatment in

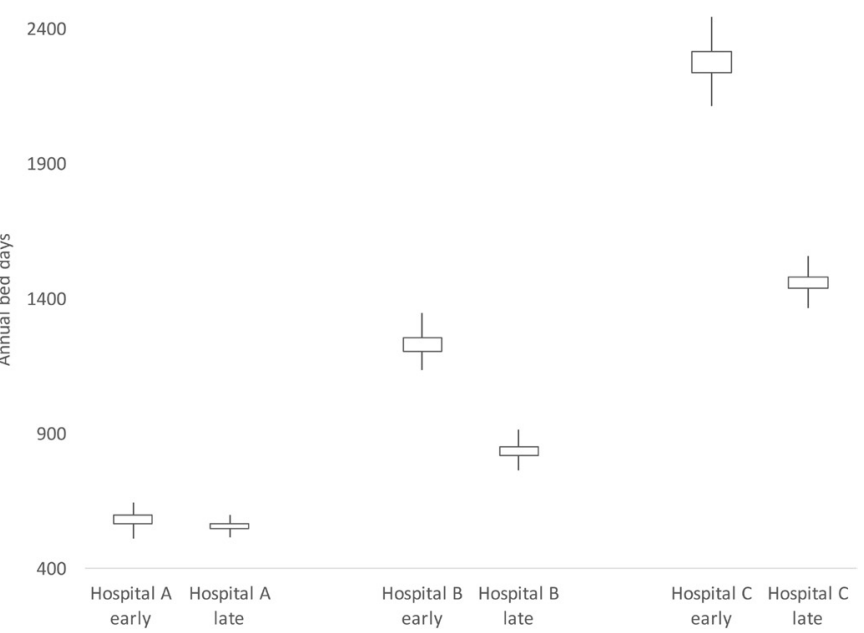

Figure 5 Annual bed days spent receiving futile treatment when applying the earliest and latest dates of futile treatment onset. Boxes depict the 25th and 75th percentile values across the bootstrapped means, vertical lines join the minimum and maximum observations. end-of-life hospital admissions, rather than a comprehensive estimate of the nature of futile treatment. There are further limitations regarding the costs assigned to futile treatment. It is naive to believe that patients would have died immediately following the onset of futile treatment, had that treatment not been provided. Instead it is likely the intensity of treatment would reduce and a transfer to a subacute or palliative care services would have arisen. These services would still incur positive costs. Thus, the costs reported here represent an upper bound on costs. This is an important caveat for those who cite the findings of this paper to argue for an investment of scarce resources for programmes that reduce futile treatment.

The nature of death has changed dramatically over the past century. Advances in the prevention of disease, as well as ongoing investment with effective healthcare interventions have improved life expectancy across the globe. Causes of death have shifted from infectious diseases towards chronic and progressive illnesses, and Australians much more commonly die in old age ${ }^{19}$ Death has become an increasingly medicalised experience and more than half of Australian deaths now occur in hospital, with $26 \%$ occurring in residential care and just $20 \%$ in the home. ${ }^{20}$

These factors, combined with an ageing population, are contributing to ever-increasing levels of healthcare resource consumption at the end of life. A recent Australian study reported that people aged 65 years and over who were in their last year of life used an estimated $10.3 \%$ of all public hospital days and accounted for $8.9 \%$ of total inpatient costs, with $40 \%$ of these costs accumulating in the last month of life. ${ }^{21}$ To ensure a sustainable healthcare system it is important that scarce resources are allocated to treatments that deliver the large patient benefit.

The findings of this study indicate that the incidence and nature of futile treatment in end-of-life admissions may differ significantly between hospitals. The impact of futile treatment is substantial in terms of both the bed days and costs expended. Yet this treatment, by definition, presents only a very low chance of achieving meaningful benefit for patients. Increased awareness of the extent of futile treatment and its impacts should stimulate the design and evaluation of interventions to reduce 
frequency. These should be tested for effectiveness, cost effectiveness and the challenges around their implementation should be documented.

Contributors All authors made substantial contributions to the conception or design of the work. MP, LB, GS and SW acquired the data. HEC, AGB and NG analysed the data. BPW, LW, LC, CG and MP interpreted the data. HEC and NG drafted the manuscript and all authors critically revised it for intellectual content. All authors gave final approval and agree to be accountable for all aspects of the work in ensuring that questions related to the accuracy or integrity of any part of the work are appropriately investigated and resolved.

Funding This research was funded by the Australian Research Council Linkage (project number LP121000096).

Competing interests None declared.

Ethics approval Multisite ethics approval for all three hospitals from the Metro South Hospital and Health Service Human Research Ethics Committee, approval number HREC/13/QPAH/651. Also obtained administrative approval from The University of Queensland Human Research Ethics Committee, approval number 2014000909 and Queensland University of Technology University Human Research Ethics Committee, approval number 1400000541 . Public Health Act approval from the Office of the Director General at the Queensland Department of Health (allows access of confidential information, given the patients were deceased and so could not consent), approval number RD005108.

Provenance and peer review Not commissioned; externally peer reviewed.

Data sharing statement The full dataset used, which has no identifying information, is available from the corresponding author.

Open Access This is an Open Access article distributed in accordance with the Creative Commons Attribution Non Commercial (CC BY-NC 4.0) license, which permits others to distribute, remix, adapt, build upon this work non-commercially, and license their derivative works on different terms, provided the original work is properly cited and the use is non-commercial. See: http://creativecommons.org/ licenses/by-nc/4.0/

(C) Article author(s) (or their employer(s) unless otherwise stated in the text of the article) 2017. All rights reserved. No commercial use is permitted unless otherwise expressly granted.

\section{REFERENCES}

1. White B, Willmott L, Close E, et al. What does "futility" mean? An empirical study of doctors' perceptions. Med J Aust 2016;204:318.

2. Moratti S. The development of "medical futility": towards a procedural approach based on the role of the medical profession. $J$ Med Ethics 2009;35:369.72.

3. Wilkinson D, Savulescu J. Knowing when to stop: futility in the intensive care unit. Current opinion in anaesthesiology 2011;24:160-5.
4. Bosslet GT, Pope TM, Rubenfeld GD, et al. An Official ATS/AACN/ ACCP/ESICM/SCCM Policy Statement: Responding to Requests for Potentially Inappropriate Treatments in Intensive Care Units. Am J Respir Crit Care Med 2015;191:1318-30.

5. Cardona-Morrell M, Kim J, Turner RM, et al. Non-beneficial treatments in hospital at the end of life: a systematic review on extent of the problem. Int J Qual Health Care 2016;28:456-69.

6. Downar J, You JJ, Bagshaw SM, et al. Nonbeneficial treatment Canada: definitions, causes, and potential solutions from the perspective of healthcare practitioners ${ }^{*}$. Crit Care Med 2015;43:270-81.

7. Rivera S, Kim D, Garone S, et al. Motivating factors in futile clinical interventions. Chest 2001;119:1944-7-7.

8. Sibbald R, Downar J, Hawryluck L. Perceptions of "futile care" among caregivers in intensive care units. CMAJ 2007;177:1201.8.

9. Huynh TN, Kleerup EC, Raj PP, et al. The opportunity cost of futile treatment in the ICU*. Crit Care Med 2014;42:1977-82.

10. Huynh TN, Kleerup EC, Wiley JF, et al. The frequency and cost of treatment perceived to be futile in critical care. JAMA Intern Med 2013;173:1887-94.

11. Sachdeva RC, Jefferson LS, Coss-Bu J, et al. Resource consumption and the extent of futile care among patients in a pediatric intensive care unit setting. J Pediatr 1996;128:742-7.

12. Highet G, Crawford D, Murray SA, et al. Development and evaluation of the Supportive and Palliative Care Indicators Tool (SPICT): a mixed-methods study. BMJ Support Palliat Care 2014;4:285-90.

13. Rechner IJ, Lipman J. The costs of caring for patients in a tertiary referral Australian Intensive Care Unit. Anaesth Intensive Care 2005:33:477-82.

14. Australian Bureau of Statistics. Consumer Price Index, Australia, Jun 2016. Canberra: Commonwealth of Australia, 2016.

15. Graves N, Harbarth S, Beyersmann J, et al. Estimating the cost of health care-associated infections: mind your p's and q's. Clin Infect Dis 2010;50:1017-21.

16. Page K, Barnett A, Graves N. Measuring the value of a hospital bed for better health care decision making. BMC Health Services Research 2017. In press.

17. Faddy M, Graves N, Pettitt A. Modeling length of stay in hospital and other right skewed data: comparison of phase-type, gamma and lognormal distributions. Value Health 2009;12:309-14.

18. Willmott $L$, White B, Gallois C, et al. Reasons doctors provide futile treatment at the end of life: a qualitative study. J Med Ethics 2016;42:496-503.

19. Swerissen H, Duckett S. Dying Well: Grattan Institute. 2014.

20. Broad JB, Gott M, Kim H, Hongsoo K, et al. Where do people die? An international comparison of the percentage of deaths occurring in hospital and residential aged care settings in 45 populations, using published and available statistics. Int $J$ Public Health 2013;58:257-67.

21. Kardamanidis K, Lim K, Da Cunha C, et al. Hospital costs of older people in New South Wales in the last year of life. Med J Aust 2007;187:383-6.

22. Australian Institute of Health and Welfare. Australian Hospital Statistics, Hospital Resources 2014-15. Health services series no 70. Canberra: AlHW 2016.

23. Australian Institute of Health and Welfare. Australian Hospital Statistics, Admitted Patient Care 2014-15. Health Services Series No 66. Canberra: AlHW 2016. 